\title{
Development of Wireless Remote Control Electric Devices for Livestock Farming Environment
}

\author{
Jianhua ZHANG ${ }^{1}$, Fantao KONG ${ }^{1}$, Zhifen $Z_{H A I}^{2}$, Shuqing HAN $^{1}$, Jing ZHANG ${ }^{1}$, Jianzhai $\mathrm{WU}^{1, * *}$ \\ ${ }^{1}$ Agricultural Information Institute of Chinese Academy of Agricultural Sciences \\ The Ministry of Agriculture Key Laboratory of Agricultural Information Service Technology \\ Beijing, 100081, China \\ ${ }^{2}$ Chinese Academy of Agricultural Engineering \\ Beijing, 100125, China \\ *E-mail: wujianzhai@caas.cn \\ $+*$ Corresponding author
}

\begin{abstract}
Suitable livestock farming environment is an important safeguard for healthy growth and high quality production of livestock. Based on the effect of greater cost-saving and efficiency- increasing of environment wireless remote control devices of the Internet of Things on livestock farming, it will greatly improve the productivity and modernization level of livestock farming. This article designs the remote control system for livestock farming environment. The system is composed of two single chip microcontrollers, power modules, DTU data transmission module, relay driver chip and relays; it adopts the dual-CPU communication mode and GPRS wireless transmission technology, also designs smartphone app program; through the wireless remote method, the device can better control the fans, heaters, lamps, motors, pumps and solenoid valves in livestock farm. The automatic control electric device of the Internet of Things combine with smart phones, tablet PCs and other mobile terminals to better adapt to the development of animal husbandry and will bring more benefits to the development of animal husbandry.
\end{abstract}

Keywords-animal husbandry; farming environment; Internet of Things; wireless remote control

\section{INTRODUCTION}

The impact of livestock growth environmental on the quantity and quality of livestock products is particularly important. At the present stage, most of livestock farms cannot control the livestock farming environment accurately, so it is difficult to further improve the quantity and quality of livestock products; and IOT technology provides the necessary path for automatic control and accurate simulation of livestock growth environment. Collect environmental information of ranch through light, temperature, humidity and gas sensors, transmit the information to the server through Wireless Sensor Network (WSN) and mobile communication technologies, such as Bluetooth, Wi-Fi, ZigBee, 3G and others; the application program compares the data collected with standard data in the database, integrates expert systems, livestock growth model and other model systems, scientifically computes the livestock farming environmental data accurately, then send the command to the terminal device, through the automatic control technology (temperature controller, light intensity controller, $\mathrm{CO} 2$ generators, etc.), carries out precise control for livestock growth environment, thereby providing a good environment for the livestock growth and promoting the improvement of quantity and quality of livestock products.

In the growth process, the growth state of individuals (such as height, weight, age, temperature, etc.) will change dramatically; for its different growth state, use feed formulations for its different growth state; carry out fine farming management for livestock so as to promote the growth more effectively and improve the quantity and quality of livestock products. In the Internet of Things for animal husbandry, through the sign index sensor for livestock, for example: pressure sensors and infrared sensors, collect the real-time data of physiological state of individuals, and transmit the data to the server in time; integrate the fine livestock feeding model to carry out scientific proportioning for livestock feed formulations, thus ensuring nutrients needed for growth of livestock, reducing costs and improving quantity and quality of livestock products; at the same time, monitor the abnormal conditions of individuals, feedback the data to producers in time and carry out real-time monitoring, feedback and processing.

Animal diseases are important factors affecting livestock quantity, particularly infectious diseases, are a great threat to livestock farming. Animal diseases have the signs before occurrence; the development of IOT technology provides technical support for monitoring and early warning of animal epidemic diseases. Through real-time monitoring of the state of individuals, know its growth state; the sensor transmits the physiological data of individuals (such as weight, body temperature, etc.) to the database through the transmission network; through monitoring of real-time data in database, the application program gets the real-time information of the growth of livestock, and compares the growth information of livestock with the latest livestock disease data, monitors the growth of livestock in time and controls the epidemic situation strictly.

\section{OVERALL DESIGN}

Transmission technology mainly means the transmission of growth environment information of livestock and conditions of the individuals to the users in wired or wireless mode. Wireless transmission technology means the use of electromagnetic signals for transmission of information, mainly including technologies of SMS communication, ZigBee wireless transmission and GPRS wireless transmission, 3G wireless communication (TD-SCDMA, WSCDMA, SCDMA2000) and 4G 
communication transmission (TD -LTE, FDD-LTE). ZigBee wireless transmission technology means to collect, summarize and transmit the farming environment information by means of the sensor nodes supporting ZigBee short-range wireless communication protocol. The protocol has the advantages of Ad Hoc Network, low cost, low power consumption and supporting of the large number of nodes. Technologies of GPRS wireless transmission, $3 \mathrm{G}$ wireless communication and $4 \mathrm{G}$ wireless transmission represent the second, third and fourth-generation of mobile communication technology respectively. GPRS wireless transmission technology has advantages of high reliability, wide coverage of signals and low cost. It is suitable for use in the condition that the involving range is wide and the layout is scattered. compared with GPRS wireless transmission technology, $3 \mathrm{G}$ wireless communication technology and $4 \mathrm{G}$ wireless transmission technology have the advantages of high data transmission rate, stable communication quality and strong real-time. In the application of animal husbandry IOT, GPRS wireless transmission technology is generally used to transmit byte data; $3 \mathrm{G}$ wireless communication technology and $4 \mathrm{G}$ wireless transmission technology are used to transmit the information of larger data volume, such as video.

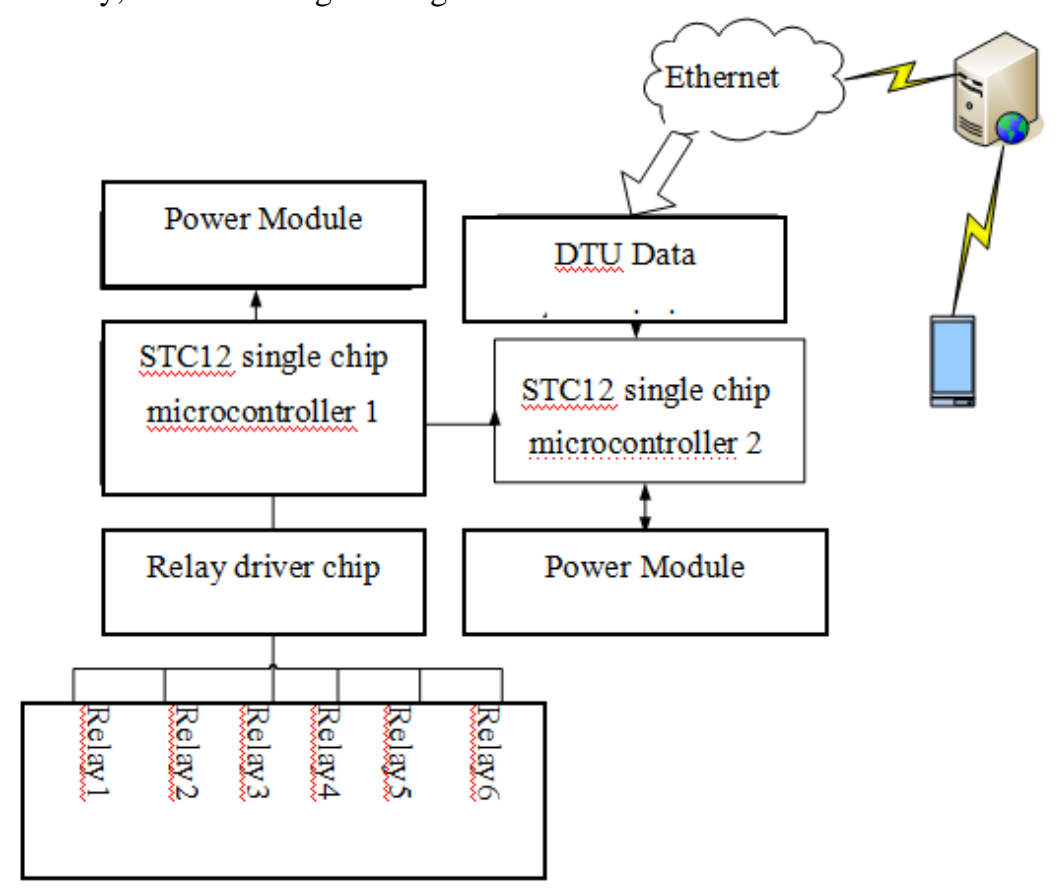

Figure 1. Hardware block diagram of the remote control system of animal husbandry IOT
Through the regulation of animal husbandry IOT equipment, the control technologies ensure the best environment for livestock farming, save production costs and reduce the burden on labor and energy consumption. They mainly include technologies of optimal control, self-adaptive control, expert control, fuzzy control, fault-tolerant control and intelligent control. The system consists of two single-chip microcomputers, power supply module, DTU data transmission module, relay driver chip and relays, as shown in Fig.1.

The optimal control technology means to determine a control method for a given system under given conditions so that the system has the best value with the prescribed performance index. The self-adaptive control technology means the control method that the system will gradually adapt and adjust itself to the optimal state through a period of operation under uncertain internal and external conditions.

Expert control technology establishes control rules and procedures on the basis of expert knowledge base; in unknown environment, imitate the experience of experts and achieve control of the system. The fuzzy control technology is to fuzzify input quantity, then develops the fuzzy control rules, outputs fuzzy decision, fuzzify output quantity and feedback; the technology is suitable for application in livestock environment; it does not require precise control, needs to save energy as much as possible and has characteristics of numerous and complex factors affecting livestock environment. Fault-tolerant control technology means the control method that the system can still maintain stability when certain components of the system fail, and can meet the requirements of certain performance index. The intelligent control technology is the most advanced automatic control. It is developed on the basis of control theory, artificial intelligence and computer science, is non-linear control and has self-learning ability.

\section{ELECTRONIC CIRCUIT DESIGN}

In order to take advantage of the developed circuit board of the logistics ranch environment meteorology and air monitoring system wireless transmission version, the remote control system uses a dual-mode communication. Single-chip microcontroller 1 is used for receiving and executing the transmitted command from single-chip microcontroller 2 and controlling the working state of relay 1-6 so as to achieve start-stop of animal husbandry IOT equipment. SCM 2 is used for receiving control commands sent by farmers via Ethernet and transmits them to single-chip microcontroller 1 . The circuit diagram 
of microcontroller 2 of the system is the same as that of the animal husbandry IOT meteorology and air monitoring system wireless transmission version. For the circuit diagram of single chip microcontroller 1 of the system, microcontroller 1 is connected with relay G5LA through the relay driver chip ULN2003. ULN2003 is the driver chip with high voltage resistance and large current, it consists of 7 silicon NPN Darlington transistors in the interior; a diode of are suppression coil back EMF is integrated in the interior, and it can be used to drive the relay. G5LA is a power relay with advantages of high reliability and big contact load, $10 \mathrm{~A}$ current and $250 \mathrm{~V}$ AC power. The wiring diagram of the remote control system PCB of animal husbandry IOT is shown in Fig.2.

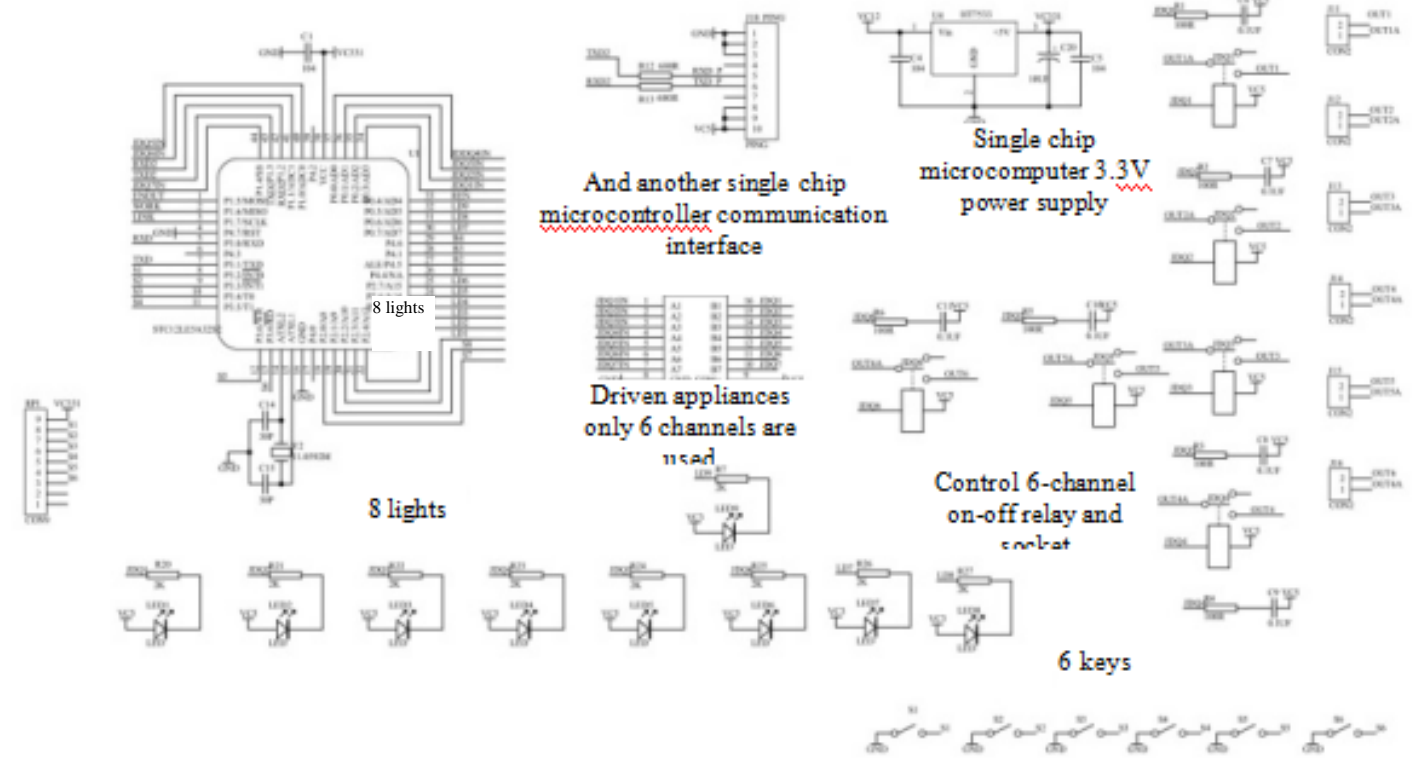

Figure 2. Remote control system circuit of animal husbandry IOT

The embedded development module is connected with sensor module through RS232 / 485 interface converter. The embedded development module is also connected to Ethernet. The embedded development module consists of S5PV210 embedded processor, memory, flash memory, Ethernet interface, RS232 interface and stabilized voltage supply module and LCD display module, as shown in
Fig.3. The embedded processor S5PV210 uses ARM CortexTM-A8 core and ARM V7 instruction set; the main frequency reaches $1 \mathrm{GHZ}$ and can achieve high-performance computing ability of 2000DMIPS (200 million instruction sets are computed per second). Memory is $512 \mathrm{MB}$ DDR2 RAM; FLASH memory is $2 \mathrm{~GB}$ MLC NAND.

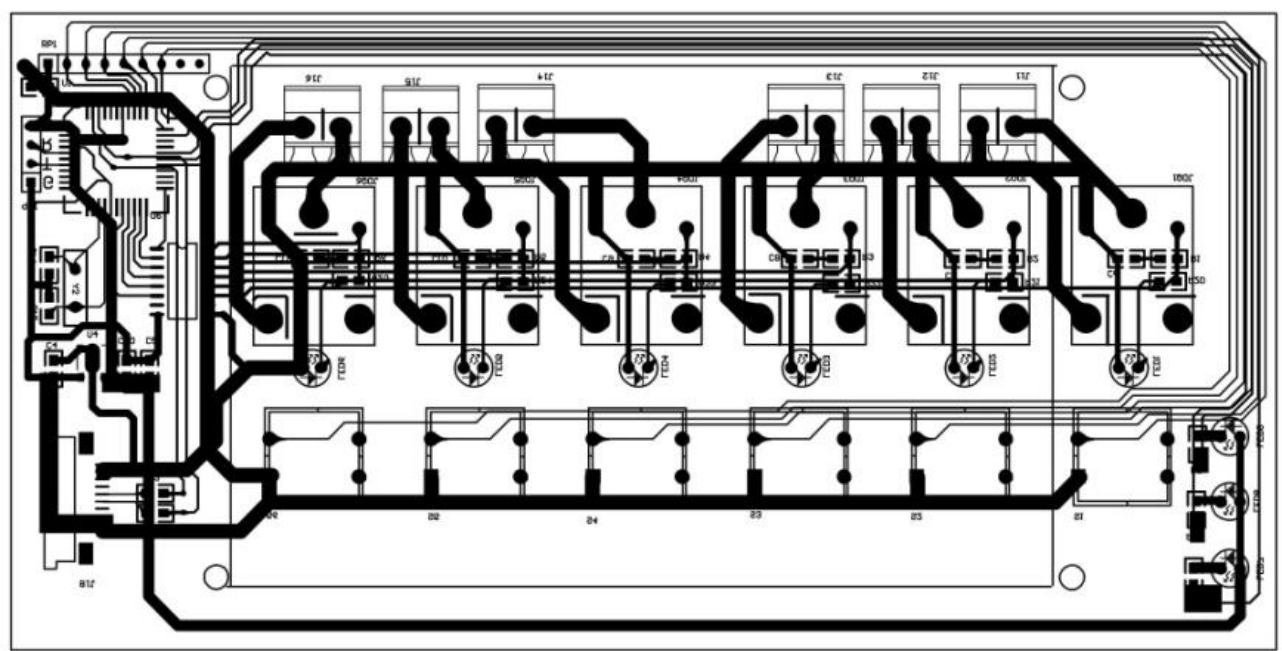

Figure 3. Remote control system circuit board PCB wiring diagram of animal husbandry IOT

\section{SYSTEM INTEGRATION}

The remote control system of the animal husbandry IOT can make farmers achieve the remote control of start and stop of ranch equipment by means of phone APP, such as fans, lamps, pumps, heaters, motors and solenoid valves. The support of remote control system of the animal husbandry IOT is shown in Figure 4. Fans, heaters, lamps, motors, pumps and solenoid valves are installed on the support. The support is easy to move and suitable for small ranch. For the control box of the remote control system of animal husbandry IOT, hardware switches of the equipment installed on the control panel can be used 
with the remote control system mobile phone APP simultaneously. For "Switch Control" module, the user can send control commands to the ranch to achieve remote control of fans, electric heaters, lamps, motors, water curtain and solenoid valves. After the equipment needing to be turned on and off is selected, click the "Send" button, as shown in Fig.4. In order to prevent disoperation, the software will prompt the user that "Are you sure to send control", as shown in Fig.6, after the user confirm again, send the control command to the server. Meanwhile, "Exit" and "Back" buttons are set at the bottom of the screen for exiting the program or returning to the function selection interface.

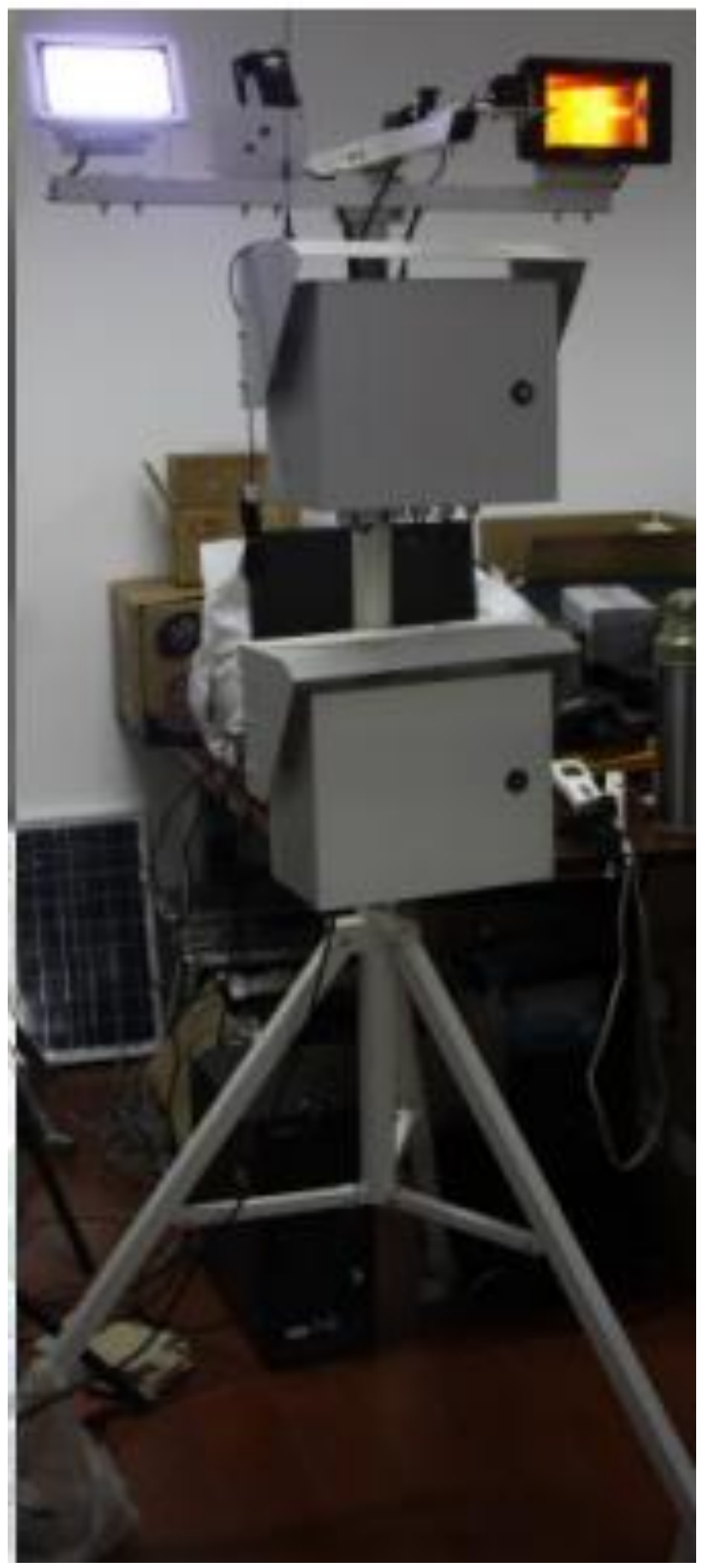

Figure 4 .

Diagram of the remote control system control device of animal husbandry IOT

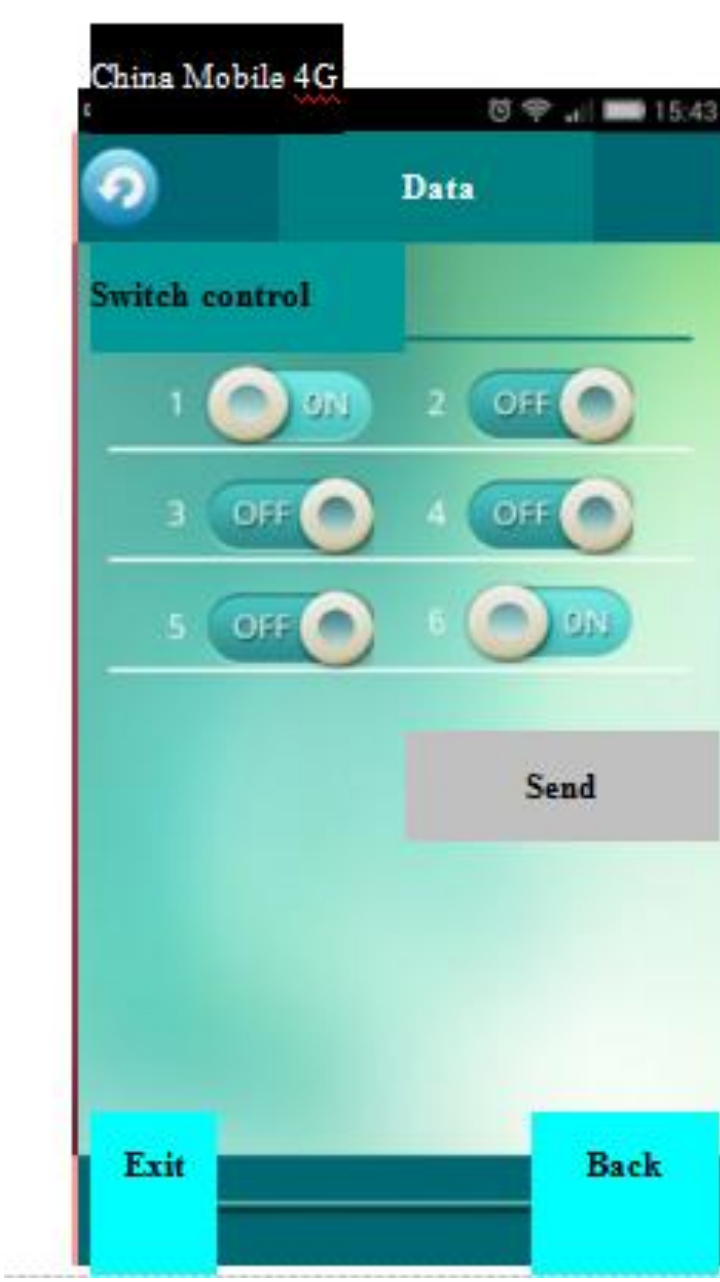

Figure 5. Remote control interface of phone APP system of animal husbandry IOT

\section{V.CONCLUSIONS}

The remote control equipment of the Internet of Things have a large role in saving cost and increasing benefit in livestock farming; they will improve the production efficiency and modernization level of livestock farming; the special equipment of IOT with low cost and high efficiency are necessary conditions for promotion and application of animal husbandry IOT. The application of IOT technology should regard the improvement of the economic efficiency of animal husbandry as the ultimate goal. Compared with the industrial and service sectors, China's agricultural industrialization level is not high and the return rate of benefit is low, so in the animal husbandry IOT in the future, the development of cost-effective and high efficiency equipment for animal husbandry IOT is the only way for the application of animal husbandry IOT. IOT application layer is combined with smart phones, tablet PCs and other mobile terminals, which has become the development characteristics of agricultural IOT. The development of special equipment for IOT more suitable for the development of animal husbandry will bring more benefits to the development of animal husbandry. 


\section{ACKNOWLEDGEMENTS}

This work is supported by Chinese Academy of Agricultural Sciences Basic research business project (Project No. Y2016PT29, Y2016PT30 and Y2016ZK17) and Chinese Academy of Agricultural Sciences Innovation Project(Project No. CAAS-ASTIP-2016-AII), and Public welfare scientific research institutes basic scientific research business special funds(Project No. JBYW-AII-2016-04, JBYW-AII-2016-04, JBYW-AII2016-09, JBYW-AII-2017-04, JBYW-AII-2017-09).

\section{REFERENCES}

[1] Application of livestock waste compost to cultivate microalgae for bioproducts production: A feasible framework[J]. Renewable and Sustainable Energy Reviews 54(2016)1285-1290.

[2] Zhu Weixing, Dai Chenyun, Huang Peng, et al. Environmental control system based on IOT for nursery pig house [J]. Transactions of the Chinese Agricultural Engineering (Transactions of the CSAE), 2012, 28(11): 177-182. (in Chinese with English abstract)

[3] Xiong Benhai, Yang Zhengang, Yang Liang, et al. Review on application of Internet of Things technology in animal husbandry in China [J]. Transactions of the Chinese Society of Agricultural Engineering (Transactions of the CSAE), 2015, 31(Supp.1): 237-246. (in Chinese with English abstract)

[4] Meng Hewei, Gao Zhenjiang, Kan Za, et al. Design and experiment on dairy cow precision-feeding device based on equal-diameter and variable-pitch[J]. Transactions of the CSAE, 2011, 27(3): 103-107. (in Chinese with English abstract)

[5] Gao Xue. Study on an Auto-Control System of Dairy Cows Recision-Feeding[D]. Beijing: China Agricultural University, 2008. (in Chinese with English abstract)

[6] Zhang Ting;Ouyang Yuanxin;Liu Yuheng,Smart Exhibition:case study of integrating RFID with wireless sensor network for pervasive computing,Dalian,China:IEEE,2008.

[7] Ivan Andonovic;Craig Michie;Michael Gilroy,Wireless Sensor Networks for Cattle Health Monitoring,Ohrid,Mar edonia:Springer,2010

[8] Yin Ling, Liu Caixing, Hong Tiansheng, et al. Design of system for monitoring dairy cattle's behavioral features based on wireless sensor networks[J]. Transactions of the CSAE, 2010, 26(3): 203-208. (in Chinese with English abstract)

[9] Nadimi E S, Sogaard H T, Bak T. ZigBee-based wireless sensor networks for classifying the behaviour of a herd of animals using classification trees[J]. Biosystems engineering, 2008, 100(2): 167-176.

[10] Karlof C, Wagner D. Secure routing in wireless sensor networks: attacks and counter measures[J]. Sensor Network Protocols and Applications, 2003, 1(2/3): 293-315. 